\title{
(2) OPEN ACCESS \\ Effect of tranexamic acid on intracranial haemorrhage and infarction in patients with traumatic brain injury: a pre-planned substudy in a sample of CRASH-3 trial patients
}

\author{
Abda Mahmood ㄷ, ' Kelly Needham, ${ }^{1}$ Haleema Shakur-Still ำ , ${ }^{1}$ Tim Harris, ${ }^{2}$ \\ Sabariah Faizah Jamaluddin (1), ${ }^{3}$ David Davies, ${ }^{4}$ Antonio Belli (1) , ${ }^{4}$ \\ Fatahul Laham Mohamed, ${ }^{5}$ Caroline Leech 우, ${ }^{6}$ Hamzah Mohd Lotfi, ${ }^{7}$ Phil Moss (i) ,

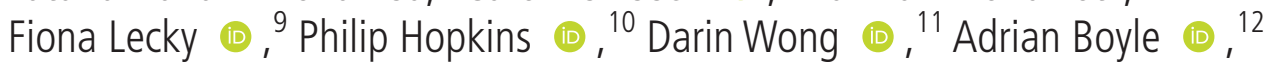 \\ Mark Wilson (1), ${ }^{13}$ Melanie Darwent (ㅇ), ${ }^{14}$ lan Roberts (i) ${ }^{1}$
}

\begin{abstract}
Handling editor Edward Carlton

Additional material is published online only. To view please visit the journal online (http://dx.doi.org/10.1136/ emermed-2020-210424).
\end{abstract}

For numbered affiliations see end of article.

\section{Correspondence to} Dr Abda Mahmood, Clinical Trials Unit, London School of Hygiene and Tropical Medicine Faculty of Epidemiology and Population Health, London WC1E 7HT, UK;

Abda.Mahmood@LSHTM.ac.uk

Received 17 July 2020 Revised 12 October 2020 Accepted 16 October 2020 Published Online First 1 December 2020

\section{Check for updates}

(C) Author(s) (or their employer(s)) 2021. Re-use permitted under CC BY. Published by BMJ.

To cite: Mahmood $A$, Needham K, ShakurStill $\mathrm{H}$, et al. Emerg Med J 2021:38:270-278.

\section{ABSTRACT}

Background Early tranexamic acid (TXA) treatment reduces head injury deaths after traumatic brain injury (TBI). We used brain scans that were acquired as part of the routine clinical practice during the CRASH-3 trial (before unblinding) to examine the mechanism of action of TXA in TBI. Specifically, we explored the potential effects of TXA on intracranial haemorrhage and infarction.

Methods This is a prospective substudy nested within the CRASH-3 trial, a randomised placebo-controlled trial of TXA (loading dose $1 \mathrm{~g}$ over $10 \mathrm{~min}$, then $1 \mathrm{~g}$ infusion over 8 hours) in patients with isolated head injury. CRASH-3 trial patients were recruited between July 2012 and January 2019. Participants in the current substudy were a subset of trial patients enrolled at 10 hospitals in the UK and 4 in Malaysia, who had at least one CT head scan performed as part of the routine clinical practice within 28 days of randomisation. The primary outcome was the volume of intraparenchymal haemorrhage (ie, contusion) measured on a CT scan done after randomisation. Secondary outcomes were progressive intracranial haemorrhage (post-randomisation CT shows $>25 \%$ of volume seen on pre-randomisation CT), new intracranial haemorrhage (any haemorrhage seen on post-randomisation (T but not on pre-randomisation CT), cerebral infarction (any infarction seen on any type of brain scan done post-randomisation, excluding infarction seen pre-randomisation) and intracranial haemorrhage volume (intraparenchymal + intraventricular + subdural + epidural) in those who underwent neurosurgical haemorrhage evacuation. We planned to conduct sensitivity analyses excluding patients who were severely injured at baseline. Dichotomous outcomes were analysed using relative risks (RR) or hazard ratios (HR), and continuous outcomes using a linear mixed model.

Results 1767 patients were included in this substudy. One-third of the patients had a baseline GCS (Glasgow Coma Score) of $3(n=579)$ and $24 \%$ had unilateral or bilateral unreactive pupils. $46 \%$ of patients were scanned pre-randomisation and postrandomisation ( $n=812 / 1767), 19 \%$ were scanned only pre-randomisation $(n=341 / 1767)$ and $35 \%$ were scanned only post-randomisation ( $n=614 / 1767)$. In all patients, there was no evidence that TXA

\section{Key messages}

What is already known on this subject

- The CRASH-3 trial showed that tranexamic acid (TXA) administered within 3 hours of injury reduced early head injury deaths in patients not at or close to the point of death. TXA treatment may have prevented deaths from post-traumatic intracranial bleeding. However, there is little evidence on the effect of TXA on intracranial bleeding as measured on CT head scans of patients with traumatic brain injury (TBI). Prior to this study, a metaanalysis of two randomised trials of TXA in TBI showed a reduction in intracranial bleeding with TXA, on CT scans that were mandated after randomisation. But because the trials were small $(n=249, n=229)$, the results were inconclusive.

\section{What this study adds}

- This substudy in 1767 CRASH-3 trial patients found that TXA may reduce intracranial bleeding, after patients with unreactive pupils at baseline were excluded. This study improves knowledge of the strengths and limitations of using routine scan data to examine the effects of a clinical trial intervention in TBI.

prevents intraparenchymal haemorrhage expansion (estimate $=1.09,95 \% \mathrm{Cl} 0.81$ to 1.45 ) or intracranial haemorrhage expansion in patients who underwent neurosurgical haemorrhage evacuation $(n=363)$ (estimate $=0.79,95 \% \mathrm{Cl} 0.57$ to 1.11 ). In patients scanned pre-randomisation and post-randomisation $(n=812)$, there was no evidence that TXA reduces progressive haemorrhage (adjusted $R R=0.91,95 \% \mathrm{Cl}$ 0.74 to 1.13 ) and new haemorrhage (adjusted $\mathrm{RR}=0.85,95 \% \mathrm{Cl} 0.72$ to 1.01$)$. When patients with unreactive pupils at baseline were excluded, there was evidence that TXA prevents new haemorrhage (adjusted $R R=0.80,95 \% \mathrm{Cl} 0.66$ to 0.98 ). In patients scanned post-randomisation $(n=1431)$, there was no evidence 
of an increase in infarction with TXA (adjusted $H R=1.28,95 \% \mathrm{Cl} 0.93$ to 1.76). A larger proportion of patients without (vs with) a postrandomisation scan died from head injury (38\% vs $19 \%$ : $R R=1.97$, $95 \% \mathrm{Cl} 1.66$ to $2.34, \mathrm{p}<0.0001$ ).

Conclusion TXA may prevent new haemorrhage in patients with reactive pupils at baseline. This is consistent with the results of the CRASH-3 trial which found that TXA reduced head injury death in patients with at least one reactive pupil at baseline. However, the large number of patients without post-randomisation scans and the possibility that the availability of scan data depends on whether a patient received TXA, challenges the validity of inferences made using routinely collected scan data. This study highlights the limitations of using routinely collected scan data to examine the effects of TBI treatments.

Trial registration number ISRCTN15088122.

\section{INTRODUCTION}

The CRASH-3 trial was a randomised placebo-controlled trial of the effects of tranexamic acid (TXA) on death and disability in patients with traumatic brain injury (TBI). ${ }^{1}$ The results showed that TXA ( $1 \mathrm{~g}$ over $10 \mathrm{~min}$ followed by $1 \mathrm{~g}$ over 8 hours) administered within 3 hours of injury reduces early head injury deaths in non-moribund patients (ie, not at or close to the point of death) (relative risk (RR) $0 \cdot 74,95 \%$ CI 0.58 to 0.94$).{ }^{23}$ While the trial was underway, the data monitoring committee asked for additional CT scan data to be collected 'to explore if, why and how patients are affected by TXA'. Consequently, we developed a separate substudy protocol to explore this aim. ${ }^{4}$ While the CRASH-3 trial was ongoing and before the results were unblinded, we examined brain scans that were acquired as part of routine clinical practice from a proportion of CRASH-3 trial patients at selected trial sites. Most TBI patients presenting to hospital have CT head scans as part of their routine clinical care. ${ }^{5}$

In this substudy, we used routinely collected scans to examine the mechanism of action of TXA in TBI, particularly the effects of TXA on intracranial bleeding and infarction. ${ }^{4} 6$ If patients who receive TXA have less intracranial bleeding or more cerebral infarction on their scans compared with those who receive placebo, this information, along with the results of the main CRASH-3 trial, could improve understanding of the mechanism of action of TXA and help generalise the CRASH-3 trial results. ${ }^{7}$

\section{METHODS}

The background, ${ }^{8}$ protocol $^{1}$ and results, ${ }^{23}$ of the CRASH-3 trial have been reported previously. Adults with head injury who were within 3 hours of injury, and had a baseline Glasgow Coma Score (GCS) of $\leq 12$ or any intracranial bleeding on CT, and no significant extracranial bleeding were eligible for randomisation in hospital. The time window for eligibility was originally 8 hours, but in 2016 the protocol was changed to limit recruitment to within 3 hours of injury. Patients with TBI were randomly allocated to receive TXA (loading dose $1 \mathrm{~g}$ over $10 \mathrm{~min}$, then $1 \mathrm{~g}$ infusion over 8 hours) or placebo ( $0.9 \%$ sodium chloride), as an additional treatment to the standard management of TBI.

Patients, care givers and those assessing outcomes were masked to allocation.

Between July 2012 and January 2019, a total of 12737 patients were randomised into the CRASH-3 trial in 175 hospitals across 29 countries, of whom 9202 patients were randomised within 3 hours of injury. This exploratory substudy (NCT01402882) was developed during the course of the CRASH-3 trial and the protocol and analysis plan were registered and published before the CRASH-3 trial was unblinded. ${ }^{46}$

Patients who fulfilled the eligibility criteria for the CRASH-3 trial were eligible for this substudy (figure 1). While this substudy was underway, we restricted its eligibility to patients with GCS $\leq 12$ since many patients with mild TBI did not have a post-randomisation scan. In addition, patients needed to have had at least one CT scan within 28 days of randomisation, and be randomised before or during the time that the single assessor of the scans was on site.

\section{Sample size}

The CRASH-2 trial substudy in 249 patients with head injury and extracranial bleeding who had one CT head scan done before randomisation and another mandated after randomisation found a $15 \%$ reduction in the volume of intracranial bleeding with TXA ( $24 \mathrm{~mL}$ TXA and $28 \mathrm{~mL}$ placebo), a correlation of 0.6 between pre-randomisation and post-randomisation bleeding volumes and a SD of $28 \mathrm{~mL} .{ }^{9}$ We used this data from the CRASH-2 substudy to estimate an unadjusted sample size of 1542 patients to achieve $80 \%$ power to detect the expected treatment effect, which was then reduced to 987 patients with adjustment for prerandomisation intracranial bleeding volume $\left(1542 \times\left(1-\left(0.6^{2}\right)\right)\right.$. We originally planned for this substudy to be conducted in 1000 trial patients, but as data collection was underway, we increased the sample size because: (1) Many patients were recruited without a pre-randomisation CT scan, and so baseline adjustment would not improve power to the extent that we expected. (2) Many patients had their post-randomisation scan done so soon after randomisation that any effect of TXA would not have sufficient opportunity to manifest on these scans, and their inclusion might dilute the treatment effect. (3) Cerebral infarction occurred less frequently than intracranial bleeding so we would not be able to reliably examine the effects of TXA on infarction. The scan assessor had time to collect data from a maximum of 2000 patients before the CRASH-3 trial completed recruitment and so this was the sample size we aimed for.

\section{Participating hospitals}

We invited CRASH-3 trial hospitals with high patient recruitment to take part in this substudy. The UK and Malaysia were two of the highest recruiting countries in the CRASH-3 trial and stored the brain images that were collected as part of the routine clinical practice on electronic systems that could be accessed in-hospital by the substudy assessor. We invited the highest recruiting hospitals from these countries to have the routinely collected brain scans of CRASH-3 trial patients examined for the purpose of this substudy. If it was possible for the substudy assessor to perform an on-site examination of the electronic brain imaging done as part of routine care at that hospital site, and the trial principal investigator at the hospital site was willing to take part, the assessor visited the site and this substudy was conducted at that site. A list of participating hospitals is shown in online supplemental appendix 1. All regulatory, ethical and local approvals were in place before data collection began.

\section{Outcome measurement}

Non-contrast enhanced CT head scans that were done prerandomisation and/or post-randomisation were examined. One outcome assessor (AM) who was blind to treatment allocation rated all scans in electronic (axial) format at each hospital using the local systems (ie, Picture Archiving and Communication System). The scans and neuroradiology reports were examined 


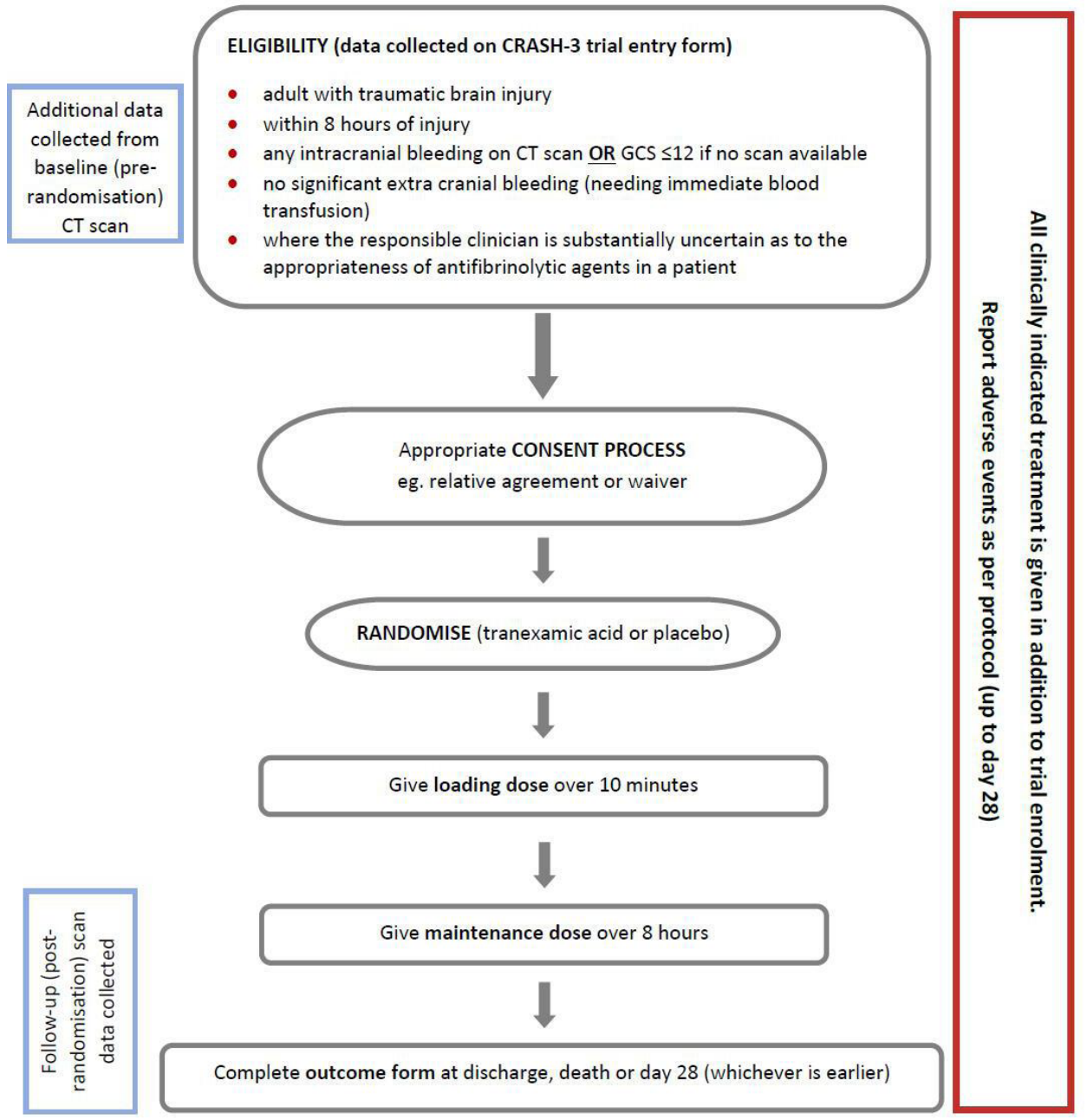

Figure 1 Flowchart: inclusion criteria for the CRASH-3 trial (blue boxes show additional procedure for the substudy). GCS,Glasgow Coma Score.

and rated using a standardised data collection form (online supplemental appendix 2).

To assess intra-rater reliability, a sample of 90 patients' scans were rated on two occasions by the same assessor, with the second reading done blind to the first. In this case, the assessor attended the same hospital site on two occasions to re-examine this sample of scans.

The CRASH-3 trial entry forms were completed by clinical research staff in participating hospitals and included a question on the type of haemorrhage seen on baseline CT. ${ }^{1}$ To examine inter-rater reliability, these CRASH-3 trial entry data that were completed by clinical research staff in participating hospitals were compared against the pre-randomisation scan readings done by the substudy outcome assessor. The second reading (substudy outcome assessor) was done blind to the first (CRASH-3 trial entry form assessors).

The ABC/2 method, a validated scale, was used to estimate the volume of epidural haemorrhage $(\mathrm{EDH})$, intraparenchymal haemorrhage (IPH) (ie, contusion) and intraventricular haemorrhage (IVH). The assessor selected the slice on which each haemorrhage was most visible. A point in the centre of the haemorrhage was found and two measurements taken: (A) the maximal diameter; (B) the width perpendicular to A. For the measurement of depth, the maximum number of slices on which the haemorrhage is visible is multiplied by slice thickness (C). These three measurements (in $\mathrm{cm}$ ) are multiplied together and then divided by 2 to estimate the volume in cubic centimetres (ie, $\mathrm{ml})$. This method assumes haemorrhage has an almost spherical shape and shows good agreement with automated volumetric analyses in IPH and EDH. ${ }^{10} 11$

The maximum thickness of subdural haemorrhage (SDH) was measured and this value substituted into a novel formula to estimate volume (see online supplemental appendix 3 ). This formula was developed by substudy collaborators at University Hospitals Birmingham (UK). ${ }^{12}$ It takes the difference between two spheres to represent the subdural space and divides this by 8 because the measurement is for unilateral SDH (divided by 2), which is typically thicker at the centre (divided by 2) and bound by superior-inferior and anterior-posterior cerebral axes (divided by 4$)$.

The volume of subarachnoid haemorrhage (SAH) cannot be estimated, and so we recorded whether or not any SAH was visible (yes/no).

\section{Primary outcome}

The primary outcome was the volume of IPH (ie, contusions) seen on the post-randomisation scan, adjusted for baseline covariates: time from injury to pre-randomisation scan, GCS score, pupil reaction, systolic blood pressure, age and participating hospital.

In the protocol, we stated that the total volume of intracranial haemorrhage would be compared between treatment groups. After publishing the protocol but before unblinding the results, ${ }^{6}$ 
we proposed (in the Statistical Analysis Plan) that any effect of TXA on intracranial haemorrhage expansion may only be reliably detected in IPH, which is less likely to require urgent neurosurgical evacuation than SDHs and EDHs. Large SDHs and EDHs may require urgent neurosurgical evacuation before we can examine any effect of TXA on the post-randomisation scans. Including these haemorrhages in the primary outcome may dilute any effect of TXA on intracranial haemorrhage expansion to the null.

\section{Primary outcome analysis plan}

A linear mixed model (LMM) was used to compare the mean change in IPH volume from pre-randomisation to postrandomisation between treatment groups. ${ }^{13}$ This model includes pre-randomisation and post-randomisation IPH volumes as correlated outcomes, with the mean post-randomisation IPH volume allowed to differ by treatment group and with the constraint of a common mean pre-randomisation IPH volume across treatment groups. ${ }^{13}$

The advantage of the LMM is that it allows all patients to be included in the analysis, even if they have missing prerandomisation or missing post-randomisation scans. The IPH volume data from all patients with a pre-randomisation scan and all patients with a post-randomisation scan is included in the analysis. This approach is less biased and more efficient than a model that could only include patients with both prerandomisation and post-randomisation scans (ie, analysis of covariance). ${ }^{13}$

\section{Secondary outcomes and analysis plan}

Progressive haemorrhage is defined as the proportion of patients with a post-randomisation scan with a total bleeding volume $(\mathrm{IPH}+\mathrm{IVH}+\mathrm{EDH}+\mathrm{SDH})$ that is more than $25 \%$ of the volume on the pre-randomisation scan. We will examine the effect of TXA on progressive haemorrhage (yes/no) using RRs and 95\% CIs. Only patients scanned both pre-randomisation and post-randomisation can be included in this analysis.

New haemorrhage is defined as the proportion of patients with any type of bleeding (IPH/IVH/EDH/SDH/SAH) on the postrandomisation scan that was not seen on the pre-randomisation scan. We will examine the effect of TXA on new haemorrhage (yes/no) using RRs and 95\% CIs. Only patients scanned both pre-randomisation and post-randomisation can be included in this analysis.

Cerebral infarction is defined as the proportion of patients with acute infarction seen on any post-randomisation brain scan done within 28 days of randomisation, excluding patients who had the same infarction on a pre-randomisation scan. All patients with a post-randomisation brain scan on which infarction could be assessed can be included in this analysis, irrespective of whether they were scanned pre-randomisation. We will examine the effect of TXA on cerebral infarction (yes/no) using HRs and 95\% CIs. HRs were used (rather than RRs) because TXA may increase cerebral infarction after a more prolonged period after randomisation and the HR accounts for the time from randomisation to the time of the scan on which infarction was seen.

Composite poor outcome is defined as the proportion of patients with progressive haemorrhage, new haemorrhage, cerebral infarction or who had neurosurgery or died due to head injury. We will examine the effect of TXA on the composite poor outcome using RRs and 95\% CIs. All substudy patients will be included in this analysis.
Volume of intracranial haemorrhage in patients who undergo surgical evacuation is defined as the total volume of intracranial haemorrhage (IPH + IVH $+\mathrm{EDH}+\mathrm{SDH})$ seen on a scan done post-randomisation and post-neurosurgery in patients who undergo neurosurgical haemorrhage evacuation. A LMM was used to examine the effect of TXA on this outcome.

All secondary outcomes will be adjusted using time from injury to pre-randomisation scan (where appropriate), GCS score, pupil reaction, systolic blood pressure, age and participating hospital.

\section{Statistical methods}

We analysed the data as per the intention-to-treat principle and using the statistical software package Stata (V.15). The analyses involved no statistical imputation. We planned to conduct sensitivity analyses excluding patients who were severely injured at baseline. We assessed the intra-rater and inter-rater reliability of dichotomous outcomes (ie, haemorrhage present/absent) and ordinal outcomes (ie, Marshall classification) using the Kappa (k) statistic, and continuous outcomes (ie, haemorrhage volume) using intraclass correlations (ICC).

\section{RESULTS}

\section{Study population at baseline}

One thousand seven hundred and sixty-seven participants recruited to the CRASH-3 trial between February 2013 and January 2019 were included in this substudy: 884 allocated to TXA group and 883 allocated to placebo group. The CONSORT (Consolidated Standards of Reporting Trials) diagram shows the disposition of patients by treatment group (figure 2) and table 1 shows their pre-randomisation characteristics.

Sixty-five per cent of the patients $(n=1147 / 1767)$ had a pre-randomisation CT scan (568 TXA group and 579 placebo group) and $71 \%$ of these patients $(\mathrm{n}=812 / 1147)$ also had a postrandomisation scan (399 TXA group and 413 placebo group). Thirty-five per cent were only scanned post-randomisation $(n=614 / 1767)$ because they had a GCS of $\leq 12$.

The median time to pre-randomisation scan was 2 hours after injury (IQR 1 hour to 2 hours). The median time to the postrandomisation scan was 23 hours after injury (IQR 8 hours to 48 hours).

Of those with a pre-randomisation scan, $97 \%$ had intracranial pathology and $82 \%$ had intracranial haemorrhage (table 2). The median volume of SDH was $46 \mathrm{~mL}$ (IQR 27 to $71 \mathrm{~mL}$ ), EDH was $6 \mathrm{~mL}$ (IQR 2 to $21 \mathrm{~mL}$ ), IPH was $1 \mathrm{~mL}$ (IQR 0.2 to $3.0 \mathrm{~mL}$ ) and IVH was $0.5 \mathrm{~mL}$ (IQR 0.2 to $1.8 \mathrm{~mL}$ ). The median volume of all haemorrhages combined was $37 \mathrm{~mL}$ (IQR 11 to $69 \mathrm{~mL}$ ).

\section{Primary outcome}

IPH volumes were skewed and so medians are presented. The median IPH volume seen after randomisation was $0.5 \mathrm{~mL}$ (IQR 0 to $4.6 \mathrm{~mL}$ ) in the TXA group and $0.3 \mathrm{~mL}$ (IQR 0 to $3.1 \mathrm{~mL}$ ) in the placebo group. Skewed data were transformed into a more normal distribution using log transformation. We used LMM to examine the effect of TXA on log-transformed IPH volumes. Covariates included age, time between injury and the prerandomisation scan, baseline GCS score and systolic blood pressure, and the participating hospital. Because IPH volumes were log-transformed, we present the anti-log of the effect estimates (and their 95\% CIs) to indicate the relative reduction or increase in IPH volume with TXA. In all patients, there was no evidence that TXA prevents IPH expansion: estimate $=1.06,95 \% \mathrm{CI}(0.84$ 


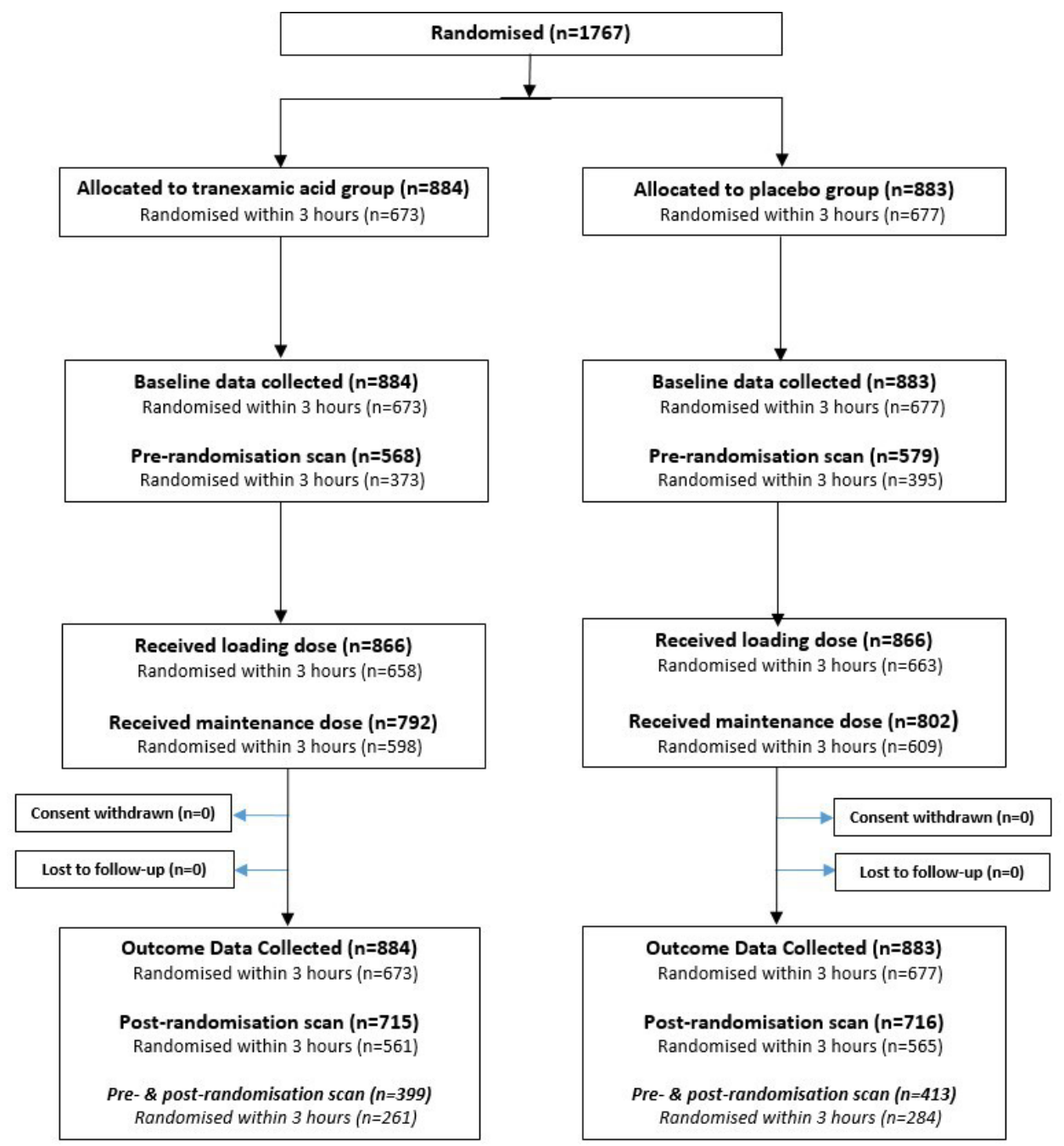

Figure 2 CONSORT (Consolidated Standards of Reporting Trials) diagram on flow of patients in the CRASH-3 trial substudy.

to 1.35$), \mathrm{p}=0.62$. This estimate cannot exclude a reduction or increase in IPH volume with TXA.

\section{Secondary outcomes}

Progressive haemorrhage. Among 812 patients scanned prerandomisation and post-randomisation, 115 (29\%) had progressive haemorrhage in the TXA group and 130 (31\%) in the placebo group (table 3$)$, adjusted $\mathrm{RR}=0.91,95 \% \mathrm{CI}(0.74$ to 1.13), $\mathrm{p}=0.41$.

New haemorrhage. Among 812 patients scanned prerandomisation and post-randomisation, 318 (39\%) had a newly detected haemorrhage on the post-randomisation scan: IPH was the most common (58\%), followed by IVH (28\%), SDH (17\%), EDH $(6 \%)$ and SAH (6\%). Fifteen percent of patients with new haemorrhage had more than one type of new haemorrhage. TXA treated patients had fewer new haemorrhages (36\% vs $42 \%$ : adjusted $\mathrm{RR}=0.85,95 \% \mathrm{CI}(0.72$ to 1.01$), \mathrm{p}=0.069)$. When the 134 patients with unilateral or bilateral unreactive pupils at baseline were excluded, the adjusted RR for a new haemorrhage was $0.80,95 \%$ CI ( 0.66 to 0.98$), \mathrm{p}=0.030$.

Cerebral infarction. Among all 1431 patients with a postrandomisation scan, $11 \%$ of patients $(n=159 / 1431)$ had an acute cerebral infarction, adjusted $\mathrm{HR}=1.28,95 \%$ (CI (0.93 to 1.76), $\mathrm{p}=0.13$.
Composite outcome. There is no evidence for a reduction in the composite with TXA $(54 \%(n=480 / 884)$ versus $54 \%$ $(\mathrm{n}=478 / 883)$ : adjusted $\mathrm{RR}=0.99,95 \% \mathrm{CI}(0.91$ to 1.07$)$, $\mathrm{p}=0.83$.

Intracranial haemorrhage in patients who underwent surgical haemorrhage evacuation. Three hundred and sixty-three patients (21\%) underwent neurosurgical haemorrhage evacuation. The median volume of haemorrhage seen after randomisation and neurosurgery was $36 \mathrm{~mL}$ (IQR 16 to $61 \mathrm{~mL}$ ) in the TXA group and $38 \mathrm{~mL}$ (IQR 18 to $70 \mathrm{~mL}$ ) in the placebo group. Haemorrhage volume data were skewed and so log-transformed. We used LMM to examine the effect of TXA on log-transformed haemorrhage volumes. Covariates included age, time between injury and the pre-randomisation scan, baseline GCS score and systolic blood pressure, and the participating hospital. We present the anti-log of the effect estimates and their 95\% CIs. There is no evidence that TXA prevents haemorrhage expansion in patients who had neurosurgical haemorrhage evacuation: estimate $=0.79,95 \%$ CI $(0.57$ to 1.11$), p=0.63$.

\section{Inter-rater and intra-rater reliability}

Inter-rater reliability for pre-randomisation haemorrhage was moderate for most haemorrhage types. EDH ratings agreed between raters in $88 \%$ of patients $(k=0.57, \mathrm{p}<0.0001)$, IVH in 
Table 1 Baseline demographic and clinical characteristics

\begin{tabular}{|c|c|c|c|}
\hline & $\begin{array}{l}\text { All patients } \\
(n=1767)\end{array}$ & $\begin{array}{l}\text { TXA group } \\
(n=884)\end{array}$ & $\begin{array}{l}\text { Placebo } \\
\text { group } \\
(n=883)\end{array}$ \\
\hline \multicolumn{4}{|l|}{ Sex } \\
\hline Male & $1413(80 \%)$ & 701 (79\%) & 712 (81\%) \\
\hline Female & $354(20 \%)$ & $183(21 \%)$ & $171(19 \%)$ \\
\hline \multicolumn{4}{|l|}{ Age } \\
\hline Median (IQR) age in years & 45 (29 to 63$)$ & 45 (29 to 64$)$ & 45 (29 to 63$)$ \\
\hline \multicolumn{4}{|l|}{ Glasgow Coma Score (GCS) } \\
\hline Mild (13 to 15$)$ & $92(5 \%)$ & $47(5 \%)$ & $45(5 \%)$ \\
\hline Moderate (9 to 12) & $532(30 \%)$ & $264(30 \%)$ & $268(30 \%)$ \\
\hline Severe (3 to 8 ) & $1143(65 \%)$ & $573(65 \%)$ & $570(65 \%)$ \\
\hline Median (IQR) GCS & 7 (3 to 10$)$ & 7 (3 to 10$)$ & 7 (3 to 10$)$ \\
\hline \multicolumn{4}{|l|}{ Pupil reaction } \\
\hline Both react & $1289(73 \%)$ & $637(72 \%)$ & $652(74 \%)$ \\
\hline One reacts & $202(11 \%)$ & $97(11 \%)$ & $105(12 \%)$ \\
\hline None react & $232(13 \%)$ & $124(14 \%)$ & $108(12 \%)$ \\
\hline Unable to assess & $43(2 \%)$ & $25(3 \%)$ & $18(2 \%)$ \\
\hline Unknown & $1(<1 \%)$ & $1(<1 \%)$ & $0(0 \%)$ \\
\hline \multicolumn{4}{|l|}{ Systolic blood pressure } \\
\hline$<90$ & $27(2 \%)$ & $14(2 \%)$ & $13(1 \%)$ \\
\hline 90 to 119 & $370(21 \%)$ & $194(22 \%)$ & $176(20 \%)$ \\
\hline$\geq 120$ & $1362(77 \%)$ & $672(76 \%)$ & $690(78 \%)$ \\
\hline Unknown & $8(<1 \%)$ & $4(<1 \%)$ & $4(<1 \%)$ \\
\hline $\begin{array}{l}\text { Median (IQR) systolic blood } \\
\text { pressure }\end{array}$ & $136(120$ to 155$)$ & 136 (120 to 156$)$ & $\begin{array}{l}136(121 \text { to } \\
154)\end{array}$ \\
\hline \multicolumn{4}{|l|}{ Hours since injury } \\
\hline$\leq 1$ & $166(9 \%)$ & $77(9 \%)$ & $89(10 \%)$ \\
\hline$>1$ to $\leq 3$ & $1184(67 \%)$ & $596(67 \%)$ & $588(67 \%)$ \\
\hline$>3$ & $417(24 \%)$ & $211(24 \%)$ & $206(23 \%)$ \\
\hline \multicolumn{4}{|l|}{ Pre-randomisation CT scan } \\
\hline Yes & $1147(65 \%)^{*}$ & $568(64 \%)$ & $579(66 \%)$ \\
\hline No & $615(35 \%)$ & $313(35 \%)$ & $302(34 \%)$ \\
\hline Unknown & $5(<1 \%)$ & $3(<1 \%)$ & $2(<1 \%)$ \\
\hline $\begin{array}{l}\text { Median (IQR) hours from } \\
\text { injury to scan }\end{array}$ & 1.8 (1.4 to 2.4$)$ & $1.8(1.5$ to 2.4$)$ & $\begin{array}{l}1.8(1.4 \text { to } \\
2.3)\end{array}$ \\
\hline
\end{tabular}

Data are $\mathrm{n}(\%)$ of patients, unless otherwise indicated.

*12 unavailable due to technical problems (4 TXA and 8 placebo).

TXA, tranexamic acid.

$88 \%(\mathrm{k}=0.50, \mathrm{p}<0.0001)$, SAH in $77 \%(\mathrm{k}=0.50, \mathrm{p}<0.0001)$, $\mathrm{SDH}$ in $74 \%(\mathrm{k}=0.43, \mathrm{p}<0.0001)$ and $\mathrm{IPH}$ in $65 \%(\mathrm{k}=0.33$, $\mathrm{p}<0.0001)$. Intra-rater reliability for haemorrhage seen prerandomisation was fair-to-moderate for most haemorrhage types. IPH ratings agreed in $65 \%$ of patients $(k=0.32, p=0.0006)$, $\mathrm{SDH}$ in $61 \%(\mathrm{k}=0.33, \mathrm{p}<0.0001)$ and $\mathrm{IVH}$ in $94 \%(\mathrm{k}=0.42$, $\mathrm{p}<0.0001)$. ICCs between individual readings for each patient (ICC $=0.70,95 \% \mathrm{CI} 0.58$ to 0.80 ) and mean readings for each patient (ICC $=0.83,95 \%$ CI 0.73 to 0.89 ) indicate moderate-togood reliability.

\section{Missing post-randomisation scans}

Three hundred and thirty-five patients did not have postrandomisation scans performed. A larger proportion of patients without (vs with) a post-randomisation scan had a mild GCS score $(8 \%$ vs $4 \%$ : $\mathrm{RR}=1.87,95 \% \mathrm{CI}(1.22$ to 2.87$), \mathrm{p}=0.004)$ or had bilateral unreactive pupils at baseline $(22 \%$ vs $11 \%$ : $\mathrm{RR}=2.04,95 \% \mathrm{CI} 1.59$ to $2.62, \mathrm{p}<0.0001$ ). A larger proportion of patients without (vs with) a post-randomisation scan died from head injury (38\% vs 19\%: RR=1.97, 95\% CI 1.66 to
Table 2 Baseline CT scan characteristics

\begin{tabular}{|cc|}
\hline & $\begin{array}{c}\text { Patients with baseline CT scan } \\
\text { ( } \mathbf{n}=1147)\end{array}$ \\
\hline Intracranial bleeding & $709(62 \%)$ \\
\hline Intraparenchymal & $184(16 \%)$ \\
\hline Intraventricular & $732(64 \%)$ \\
\hline Subdural & $215(19 \%)$ \\
\hline Epidural & $829(72 \%)$ \\
\hline Subarachnoid & $177(15 \%)$ \\
\hline Oedema and infarction & $13(1 \%)$ \\
\hline Oedematous lesions & \\
\hline Acute cerebral infarction & $503(44 \%)$ \\
\hline Mass effect & $6(4$ to 12$)$ \\
\hline Midline shift & $609(53 \%)$ \\
\hline Median (IQR) degree of shift in mm & $456(40 \%)$ \\
\hline Sulcal effacement & \\
\hline Ventricular effacement & $25(2 \%)$ \\
\hline Marshall classification & $533(46 \%)$ \\
\hline Diffuse injury I & $60(5 \%)$ \\
\hline Diffuse injury II & $30(3 \%)$ \\
\hline Diffuse injury III & $487(43 \%)$ \\
\hline Diffuse injury IV & $12(1 \%)$ \\
\hline Non-evacuated mass lesion & \\
\hline Unknown & \\
\hline Data ar n & \\
\hline
\end{tabular}

Data are $\mathrm{n}(\%)$ of patients, unless otherwise indicated

2.34, p<0.0001) (tables 4 and 5). There was no evidence that TXA increases the risk of not having a post-randomisation scan: $19 \%(n=169 / 884)$ versus $19 \%(n=166 / 882), R R=1.02,95 \% C I$ (0.84 to 1.23$), \mathrm{p}=0.87$.

Outcomes are presented separately for patients randomised within 3 hours of injury and patients randomised after 3 hours of injury (table 3 and online supplemental appendix 4).

\section{DISCUSSION}

\section{Substudy findings in context of CRASH-3 trial results}

The CRASH-3 trial showed that TXA reduces early head injury deaths in non-moribund patients. ${ }^{23}$ In this substudy, we attempted to obtain more information about the mechanism of TXA by looking at the brain scans of patients in each trial arm. Performing brain scans was not a requirement of the CRASH-3 trial protocol and so we reviewed clinically indicated scans from a subset of trial patients. Using the date and time of the scans, and the date and time of randomisation into the trial, we were able to confirm whether clinically indicated scans were done before, after, or both before and after randomisation. We did not find evidence for a difference in the volume of intracranial bleeding between TXA and control patients on scans done postrandomisation. Comparing the scans of patients who had both pre-randomisation and post-randomisation scans, we did not find evidence for a reduction in intracranial bleeding with TXA. We did find that the TXA group had fewer new haemorrhages, if we excluded patients whose baseline clinical exam indicated one or both non-reactive pupils.

However, because a large proportion of patients with milder injuries were not scanned after randomisation, we were unable to examine the effect of TXA in the population most likely to benefit. The majority of patients in this substudy had severe head injury and one-third had a GCS score of 3. This was partly because we chose high recruiting hospitals to participate in this substudy, many of which were also major trauma centres, which 
Table 3 Effect of TXA on new and progressive haemorrhage

\begin{tabular}{|c|c|c|c|c|c|c|}
\hline & TXA group & Placebo group & RR $(95 \% \mathrm{Cl})$ unadjusted & $\begin{array}{l}\text { P value } \\
\text { (two-tailed) }\end{array}$ & RR $(95 \% \mathrm{Cl})$ adjusted ${ }^{*}$ & $\begin{array}{l}\text { P value } \\
\text { (two-tailed) }\end{array}$ \\
\hline \multicolumn{7}{|l|}{ New haemorrhage } \\
\hline$\leq 3$ hours since injury & 108/261 (41\%) & 129/284 (45\%) & $0.91(0.75$ to 1.10$)$ & 0.34 & 0.89 (0.73 to 1.08$)$ & 0.22 \\
\hline All patients & $144 / 399(36 \%)$ & $174 / 413(42 \%)$ & $0.86(0.72$ to 1.02$)$ & 0.079 & 0.85 (0.72 to 1.01$)$ & 0.069 \\
\hline Exclude unreactive pupils (one/both) & $108 / 332(33 \%)$ & $140 / 346(40 \%)$ & 0.80 (0.66 to 0.98$)$ & 0.033 & 0.80 (0.66 to 0.98$)$ & 0.030 \\
\hline$>3$ hours since injury & $39 / 138(28 \%)$ & $42 / 129(33 \%)$ & $0.87(0.60$ to 1.25$)$ & 0.45 & 0.86 (0.58 to 1.28$)$ & 0.46 \\
\hline All patients & $115 / 399(29 \%)$ & $130 / 413(31 \%)$ & $0.92(0.74$ to 1.13$)$ & 0.41 & 0.91 (0.74 to 1.13$)$ & 0.41 \\
\hline Exclude unreactive pupils (one/both) & $92 / 332(28 \%)$ & $111 / 346(32 \%)$ & 0.86 (0.69 to 1.09$)$ & 0.22 & $0.87(0.69$ to 1.10$)$ & 0.24 \\
\hline
\end{tabular}

${ }^{*}$ Adjusted for time from injury to pre-randomisation scan, age, GlasgowComa Score, pupil reaction, systolic blood pressure and participating hospital.

$\mathrm{RR}$, relative risk; TXA, tranexamic acid.

receive a large proportion of severely injured patients. The CRASH-3 trial found a reduction in head injury death within 24 hours of injury in non-moribund patients. ${ }^{23}$ Given the baseline status of the included patients, it would be difficult to see a treatment effect on CT scans in this substudy, which included a large proportion of moribund patients. The baseline data show that patients with severe TBI and unreactive pupils have the largest volumes of intracranial bleeding. ${ }^{14}$ TXA may have little potential to prevent intracranial haemorrhage progression in these patients, and their inclusion may dilute any treatment effect. In fact, much like the CRASH-3 trial which found a reduction in head injury death when patients with unreactive pupils were excluded, ${ }^{2}{ }^{3}$ when those with unreactive pupils are excluded in this substudy, there is some evidence that TXA prevents new haemorrhage.

\section{Strengths and weaknesses in relation to other studies}

With a limited clinical trial budget, we had to rely on scans that were ordered by treating physicians as part of trial patients' clinical management. This allowed us to conduct the largest mechanistic study of TXA in TBI to date. However, there are methodological flaws associated with the use of routine imaging. Previous trials in this area were smaller and mandated

Table 4 Patients with post-randomisation scans by baseline injury severity and treatment group

\begin{tabular}{|c|c|c|c|c|c|}
\hline & $\begin{array}{l}\text { Patients with post- } \\
\text { randomisation scan } \\
\text { ( } \mathrm{n}=1431 / 1767)\end{array}$ & TXA group & Placebo group & RR $(95 \% \mathrm{Cl})$ & $\begin{array}{l}\text { P value } \\
\text { (two-tailed) }\end{array}$ \\
\hline \multicolumn{6}{|l|}{ Glasgow Coma Score (GCS) } \\
\hline Mild (GCS 13 to 15) & $64(4 \%)$ & $32 / 47(68 \%)$ & $32 / 45(71 \%)$ & 0.96 (0.73 to 1.26$)$ & 0.75 \\
\hline Moderate (GCS 9 to 12) & $425(30 \%)$ & $211 / 264(80 \%)$ & $214 / 267(80 \%)$ & $1.00(0.92$ to 1.09$)$ & 0.95 \\
\hline Severe (GCS 3 to 8 ) & $942(66 \%)$ & $472 / 573(82 \%)$ & $470 / 570(82 \%)$ & 1.00 (0.95 to 1.05$)$ & 0.97 \\
\hline \multicolumn{6}{|l|}{ Pupil reaction } \\
\hline Both react & $1064(74 \%)$ & $529 / 637(83 \%)$ & $535 / 651(82 \%)$ & 1.01 (0.96 to 1.06$)$ & 0.68 \\
\hline One reacts & $174(12 \%)$ & $83 / 97(86 \%)$ & $91 / 105(87 \%)$ & 0.99 (0.88 to 1.10$)$ & 0.82 \\
\hline None react & $157(11 \%)$ & $82 / 124(66 \%)$ & $75 / 108(69 \%)$ & 0.95 (0.80 to 1.14$)$ & 0.59 \\
\hline Unable to assess & $35(2 \%)$ & $20 / 25(80 \%)$ & $15 / 18(83 \%)$ & 0.96 (0.72 to 1.28$)$ & 0.78 \\
\hline
\end{tabular}

$\mathrm{RR}$, relative risk; TXA, tranexamic acid.

Table 5 Patients without post-randomisation scans by baseline injury severity and treatment group

\begin{tabular}{llcccc}
\hline & $\begin{array}{l}\text { Patients without post-randomisation scan } \\
(\mathbf{n}=335 / 1767)\end{array}$ & TXA group & Placebo group & RR (95\% Cl) & $\begin{array}{l}\text { P value } \\
\text { (two-tailed) }\end{array}$ \\
\hline Glasgow Coma Score (GCS) & & & & & \\
\hline Mild (GCS 13 to 15) & $28(8 \%)$ & $15 / 47(32 \%)$ & $13 / 45(29 \%)$ & $1.10(0.59$ to 2.06) & 0.75 \\
\hline Moderate (GCS 9 to 12) & $106(32 \%)$ & $53 / 264(20 \%)$ & $53 / 267(20 \%)$ & $1.01(0.72$ to 1.42$)$ & 0.95 \\
\hline Severe (GCS 3 to 8) & $201(60 \%)$ & $101 / 573(18 \%)$ & $100 / 570(18 \%)$ & $1.00(0.78$ to 1.29$)$ & 0.97 \\
\hline Pupil reaction & & & & & \\
\hline Both react & $224(67 \%)$ & $108 / 637(17 \%)$ & $116 / 651(18 \%)$ & $0.95(0.75$ to 1.21$)$ & 0.68 \\
\hline One reacts & $28(8 \%)$ & $14 / 97(14 \%)$ & $14 / 105(13 \%)$ & $1.08(0.54$ to 2.16$)$ & 0.82 \\
\hline None react & $75(22 \%)$ & $42 / 124(34 \%)$ & $33 / 108(31 \%)$ & $1.11(0.76$ to 1.62$)$ & 0.59 \\
\hline Unable to assess & $8(2 \%)$ & $5 / 25(20 \%)$ & $3 / 18(17 \%)$ & $1.20(0.32$ to 4.46$)$ & 0.79 \\
\hline
\end{tabular}

$\mathrm{RR}$, relative risk; TXA, tranexamic acid 
scans post-randomisation. ${ }^{9} 1516$ They had fewer missing postrandomisation scans and in this respect are less vulnerable to bias.

\section{Substudy weaknesses}

Although using data collected as part of standard practice in clinical trials can reduce costs, there are threats to validity. ${ }^{17} 18 \mathrm{In}$ the context of TBI, an initial or repeat CT brain scan may not be performed in patients with mild or unsurvivable injury, and if a second scan is clinically indicated, patients may die before it can be conducted. These missing scans threaten the validity of any inferences. A recent systematic review revealed that there is substantial variability in how missing data are handled in TBI research. ${ }^{19}$ If there is minimal missing data, the impact on effect estimates can be negligible. ${ }^{20}$ In this substudy, one-fifth of the patients did not receive a post-randomisation CT brain scan, which may be related to their prognosis and/or response to TXA. ${ }^{21}$ Because TXA reduces the risk of head injury death, ${ }^{2}$ TXA-treated patients may not have had a clinical indication for a post-randomisation scan and placebo-treated patients may have died before the opportunity for scanning. Therefore, a similar proportion of patients with missing post-randomisation scans in TXA and placebo groups does not exclude the possibility of bias.

One-fifth of the patients had neurosurgical haemorrhage evacuation before the post-randomisation scan. Haemorrhage seen post-randomisation and post-neurosurgery may reflect the combined effects of TXA and neurosurgical haemorrhage evacuation. If TXA reduces intracranial haemorrhage, it may reduce the need for neurosurgical haemorrhage evacuation. Patients who receive placebo and go on to have their intracranial bleed evacuated may have less blood on their post-randomisation scan than those who receive TXA and do not undergo evacuation.

The clinical value of an arbitrarily defined progressive haemorrhage outcome is limited. An apparent increase in haemorrhage between scans may not be generalisable because this may have different clinical implications depending on the type of haemorrhage that expands. Even though $\mathrm{SDH} / \mathrm{EDH}$ are typically larger than IPH/IVH, a 25\% increase in SDH/EDH could be managed surgically in the first few hours of injury with good prognostic outcome, ${ }^{22}$ but a $25 \%$ increase in IPH/IVH may have worse prognostic outcome. ${ }^{23}$

Outcomes may not have been accurately measured. The ABC/2 method has not been validated in all types of haemorrhage (ie, IVH) and its accuracy is reduced if bleeds are irregularly shaped. SDH volume estimation was based on a novel approach whose reliability is to be confirmed. Unclotted bleeding, micro-bleeding and infarction are not clearly visible on CT, especially soon after injury. ${ }^{24-26}$ Furthermore, reliability ratings indicate that there were discrepancies in ratings between and within assessors. Discrepancies between raters should be expected because the substudy assessor examined scans and radiology reports that are usually written post-randomisation, while randomisation into the CRASH-3 trial was based on information known pre-randomisation (eg, verbal report from clinician). Discrepancies in the substudy assessor's measurements of the same scans should be expected because Reading 1 was done as part of an audit which primarily involved estimating the volume of the largest bleeds, while Reading 2 was done as part of the substudy procedure and examined all bleeds.

There were two sources of null bias. First, many postrandomisation scans were done very soon after randomisation, when there would have been little opportunity for any effect of TXA to become apparent. This is particularly relevant for types of haemorrhage that usually have smaller volumes, such as IPH, which the baseline data suggest expand at $0.4 \mathrm{~mL} /$ hour (IQR 0.1 to $2 \mathrm{~mL} /$ hour). ${ }^{14}$ Second, a large proportion of patients had severe (and possibly unsurvivable) head injuries at baseline. Because the CRASH-3 trial did not find evidence for a reduction in head injury death in severely injured patients, their inclusion in this substudy may have also diluted any treatment effect.

\section{Implications for future research}

The US National Institutes of Health propose that 'the link between prevention of haemorrhage growth (with haemostatic therapy) and clinical outcome' can be studied using radiological outcomes. ${ }^{27}$ From our experience of conducting this substudy, we believe that this should be done in a large high-quality randomised trial where all randomised patients are scanned post-randomisation. Inclusion should not be based on postrandomisation events or restricted in terms of injury severity. MRI is more sensitive than CT in detecting micro-bleeding ${ }^{25}$ and infarction. $^{26}$

\section{CONCLUSION}

This substudy was nested within the CRASH-3 trial and its results are consistent with the trial results. Here we found that TXA may prevent new haemorrhage in patients with reactive pupils at baseline, and the CRASH-3 trial found that TXA reduced head injury death in patients with at least one reactive pupil at baseline. While the use of routinely collected scans allowed this substudy to achieve a sample size that is considerably larger than previous studies in this area, this also resulted in many methodological flaws. Most importantly, the large number of patients without post-randomisation scans and the possibility that the availability of scan data depends on whether a patient received TXA, challenges the validity of treatment effect estimates. This study highlights the strengths and limitations of using routinely collected scan data to examine the effects of TBI treatments.

\section{Author affiliations}

${ }^{1}$ Clinical Trials Unit, London School of Hygiene \& Tropical Medicine Faculty of Epidemiology and Population Health, London, UK

${ }^{2}$ Department of Emergency Medicine, Royal London Hospital, Barts Health NHS Trust, London, UK

${ }^{3}$ Emergency Department, Hospital Sungai Buloh, Sungai Buloh, Malaysia

${ }^{4} \mathrm{NIHR}$ Surgical Reconstruction and Microbiology Research Centre, University

Hospitals Birmingham NHS Foundation Trust, Birmingham, UK

${ }^{5}$ Emergency Department, Hospital Sultanah Bahiyah, Alor Setar, Malaysia

${ }^{6}$ Emergency Department, University Hospitals Coventry \& Warwickshire NHS Trust, Coventry, UK

${ }^{7}$ Emergency Department, Hospital Sultanah Nur Zahirah, Kuala Terengganu, Malaysia ${ }^{8}$ Clinical Research Unit, Emergency Department, Saint George's University Hospitals NHS Foundation Trust, London, UK

${ }^{9}$ Accident \& Emergency, Salford Royal NHS Foundation Trust, Salford, UK

${ }^{10}$ Emergency Department, King's College Hospital NHS Foundation Trust, London, UK

${ }^{11}$ Emergency Department, Penang General Hospital, Georgetown, Malaysia

${ }^{12}$ Emergency Department, Addenbrooke's Hospital Cambridge University Hospitals NHS Foundation Trust, Cambridge, UK

${ }^{13}$ Neurosurgeries, Emergencies \& Trauma, Division of Medicine, St Mary's Hospital, Imperial College Healthcare NHS Trust, London, UK

${ }^{14}$ Emergency Department, Oxford University Hospitals NHS Foundation Trust, Oxford, UK

Twitter Abda Mahmood @AbdaMahmood, Caroline Leech @leechcaroline and Adrian Boyle @dradrianboyle

Acknowledgements The authors are grateful to all patients who took part in this study, the Trial Co-ordinating Centre at the Clinical Trials Unit (London School of Hygiene \& Tropical Medicine, LSHTM), Medical Statisticians at LSHTM for their expert advice and the clinical research staff at each participating hospital for facilitating this study (see Appendix 1 for list of participating hospitals). 
Contributors AM drafted and revised this manuscript. She led the protocol development and amendments, national and international ethics and regulatory submissions and amendments, local hospital site approvals and updates, data collection, data and database management, data cleaning, analysis, interpretation and publication, of this study. KN provided expertise in statistical analysis. HS-S facilitated protocol development, study design and conduct. DD provided guidance in rating CT scans and developing the method. TH, SFJ, AB, FLM, CL, MLH, PM, FLM, PH, $D W, A B, M W$ and MD facilitated data collection at their hospital sites. IR provided epidemiological oversight from conception to publication. All authors critically read the manuscript and provided feedback for improvement.

Funding This study is fully funded by the London School of Hygiene \& Tropical Medicine (Grant reference EPAA6020). The design, management and interpretation of this study is entirely independent of the manufacturers of TXA and the funders.

Competing interests All authors have completed the ICMJE uniform disclosure form and declare: AM received a PhD Studentship from the Clinical Trials Unit (LSHTM) from September 2015 to February 2020; A Belli received grants from the Medical Research Council (MRC) and National Institute for Health Research (NIHR) during the conduct of this study; $\mathrm{CL}$ is an Associate Editor for the Emergency Medicine Journal; no other relationships or activities that could appear to have influenced the submitted work.

Patient consent for publication Not required.

Ethics approval The Medical Research and Ethics Committee and Health Research Authority reviewed the protocol and supporting documents for this study and provided a favourable ethical opinion on 8 June 2016 (Research Ethics Committee Reference 12/EE/0274). All participating hospitals provided local approvals and letters of access for this study to be conducted at their respective sites. Favourable ethical opinion was received from the Observational/Interventions Research Ethics Committee at the London School of Hygiene \& Tropical Medicine on 24 May 2016 (Reference 11535).

Provenance and peer review Not commissioned; externally peer reviewed.

Data availability statement Data are available upon reasonable request. Following the publication of the analyses detailed in the study SAP, individual deidentified patient data, including a data dictionary, will be made available via our data-sharing portal, The Free Bank of Injury and Emergency Research Data (freeBIRD) website (https://freebird.Lshtm.ac.uk) indefinitely. This will allow for maximum utilisation of the data to improve patient care and advance knowledge. The study protocol, SAP and results will be published open-access and freely available at https://www.txacentral.org/. If additional analyses are proposed, we would request a protocol and expect that a data access agreement is in place.

Supplemental material This content has been supplied by the author(s). It has not been vetted by BMJ Publishing Group Limited (BMJ) and may not have been peer-reviewed. Any opinions or recommendations discussed are solely those of the author(s) and are not endorsed by BMJ. BMJ disclaims all liability and responsibility arising from any reliance placed on the content. Where the content includes any translated material, BMJ does not warrant the accuracy and reliability of the translations (including but not limited to local regulations, clinical guidelines, terminology, drug names and drug dosages), and is not responsible for any error and/or omissions arising from translation and adaptation or otherwise.

Open access This is an open access article distributed in accordance with the Creative Commons Attribution 4.0 Unported (CC BY 4.0) license, which permits others to copy, redistribute, remix, transform and build upon this work for any purpose, provided the original work is properly cited, a link to the licence is given, and indication of whether changes were made. See: https://creativecommons.org/ licenses/by/4.0/.

\section{ORCID iDs}

Abda Mahmood http://orcid.org/0000-0002-3267-4590

Haleema Shakur-Still http://orcid.org/0000-0002-6511-109X

Sabariah Faizah Jamaluddin http://orcid.org/0000-0002-3788-7915

Antonio Belli http://orcid.org/0000-0002-3211-9933

Caroline Leech http://orcid.org/0000-0002-5301-9798

Phil Moss http://orcid.org/0000-0002-5551-6261

Fiona Lecky http://orcid.org/0000-0001-6806-0921

Philip Hopkins http://orcid.org/0000-0002-3713-8391

Darin Wong http://orcid.org/0000-0003-3784-4621

Adrian Boyle http://orcid.org/0000-0002-9009-5423

Mark Wilson http://orcid.org/0000-0002-2346-4911

Melanie Darwent http://orcid.org/0000-0003-0600-0020

Ian Roberts http://orcid.org/0000-0003-1596-6054

\section{REFERENCES}

1 Dewan Y, Komolafe EO, Mejía-Mantilla JH, et al. CRASH-3 - tranexamic acid for the treatment of significant traumatic brain injury: study protocol for an international randomized, double-blind, placebo-controlled trial. Trials 2012;13:87.

2 CRASH-3 trial collaborators. Effects of tranexamic acid on death, disability, vascular occlusive events and other morbidities in patients with acute traumatic brain injury (CRASH-3): a randomised, placebo-controlled trial. Lancet 2019;394:1713-23.

3 Brenner A, Belli A, Chaudhri R, et al. Understanding the neuroprotective effect of tranexamic acid: an exploratory analysis of the CRASH-3 randomised trial. BMC Critical Care 2020

4 Mahmood A, Roberts I, Shakur H. A nested mechanistic sub-study into the effect of tranexamic acid versus placebo on intracranial haemorrhage and cerebral ischaemia in isolated traumatic brain injury: study protocol for a randomised controlled trial (CRASH-3 Trial Intracranial Bleeding Mechanistic Sub-Study [CRASH-3 IBMS]). Trials 2017; 18:330.

5 Davis T, Ings A, National Institute of Health and Care Excellence. Head injury: triage, assessment, investigation and early management of head injury in children, young people and adults (NICE guideline CG 176). Arch Dis Child Educ Pract Ed 2015:100:97-100.

6 Mahmood A, Roberts I, Shakur-Still H. A nested randomised trial of the effect of tranexamic acid on intracranial haemorrhage and infarction in traumatic brain injury (CRASH-3 trial intracranial bleeding mechanistic study): statistical analysis plan. Wellcome Open Res 2018;3:99.

7 Roberts I, Prieto-Merino D. Applying results from clinical trials: tranexamic acid in trauma patients. J Intensive Care 2014;2:56.

8 Mahmood A, Roberts I, Shakur H, et al. Does tranexamic acid improve outcomes in traumatic brain injury? BMJ 2016;354:i4814.

9 Perel P, Al-Shahi Salman R, Kawahara T, et al. CRASH-2 (Clinical Randomisation of an Antifibrinolytic in Significant Haemorrhage) intracranial bleeding study: the effect of tranexamic acid in traumatic brain injury-a nested randomised, placebo-controlled trial. Health Technol Assess 2012;16:iii-xii, 1-54.

10 Webb AJS, Ullman NL, Morgan TC, et al. Accuracy of the ABC/2 score for intracerebral hemorrhage: systematic review and analysis of MISTIE, CLEAR-IVH, and clear III. Stroke 2015:46:2470-6.

11 Hu T-T, Yan L, Yan P-F, et al. Assessment of the ABC/2 method of epidural hematoma volume measurement as compared to computer-assisted planimetric analysis. Biol Res Nurs 2016:18:5-11.

12 Su Z, Wang M, Davies D, et al. Preliminary results of the Golden Hour study - Part II: Multi-dimensional outcomes of traumatic brain injury in comparison with orthopaedic controls [abstract]. European Association of Neurosurgical Societies 2006.

13 Liu GF, Lu K, Mogg R, et al. Should baseline be a covariate or dependent variable in analyses of change from baseline in clinical trials? Stat Med 2009;28:2509-30.

14 Mahmood A, Needham K, Shakur-Still H, et al. Tranexamic acid in traumatic brain injury: an explanatory study nested within the CRASH-3 trial. European Journal of Trauma and Emergency Surgery 2020.

15 Yutthakasemsunt S, Kittiwatanagul W, Piyavechvirat P, et al. Tranexamic acid for patients with traumatic brain injury: a randomized, double-blinded, placebo-controlled trial. BMC Emerg Med 2013;13:20.

16 Fakharian E, Abedzadeh-Kalahroudi M, Atoof F. Effect of tranexamic acid on prevention of hemorrhagic mass growth in patients with traumatic brain injury. World Neurosurg 2018;109:e748-53.

17 Williams JG, Cheung WY, Cohen DR, et al. Can randomised trials rely on existing electronic data? A feasibility study to explore the value of routine data in health technology assessment. Health Technol Assess 2003;7:1-117.

18 Cook JA, Collins GS. The rise of big clinical databases. Br J Surg 2015;102:e93-101.

19 Richter S, Stevenson S, Newman T, et al. Handling of missing outcome data in traumatic brain injury research: a systematic review. J Neurotrauma 2019;36:2743-52.

20 Jakobsen JC, Gluud C, Wetterslev J, et al. When and how should multiple imputation be used for handling missing data in randomised clinical trials - a practical guide with flowcharts. BMC Med Res Methodol 2017;17:162.

21 Altman DG. Missing outcomes in randomized trials: addressing the dilemma. Open Med 2009;3:e51-3.

22 Haselsberger K, Pucher R, Auer LM. Prognosis after acute subdural or epidural haemorrhage. Acta Neurochir 1988;90:111-6.

23 Hinson HE, Hanley DF, Ziai WC. Management of intraventricular hemorrhage. Curr Neurol Neurosci Rep 2010;10:73-82.

24 Parizel PM, Makkat S, Van Miert E, et al. Intracranial hemorrhage: principles of $C T$ and MRI interpretation. Eur Radiol 2001;11:1770-83

25 Haller S, Vernooij MW, Kuijer JPA, et al. Cerebral microbleeds: imaging and clinical significance. Radiology 2018;287:11-28.

26 Tatlisumak T. Is CT or MRI the method of choice for imaging patients with acute stroke? Why should men divide if fate has United? Stroke 2002;33:2144-5.

27 Hemostasis Clinical Trial Outcomes Working Group. Hemostasis outcomes for clinical trials workshop, 2019. Available: https://www.evensi.us/hemostasis-clinical-trialsworking-group-nih-building-45/314238921 